\title{
Modeling brain functional networks using logic relationships Jian $\mathrm{Li}^{1}$ and Yiyuan Tang*1,2
}

\author{
Address: ${ }^{1}$ Institute of Neuroinformatics and Laboratory for Brain and Mind, Dalian University of Technology, Dalian 116024, PR China and \\ ${ }^{2}$ Department of Psychology, University of Oregon, Eugene, OR 97403, USA \\ Email: Yiyuan Tang* - yiyuan@uoregon.edu \\ * Corresponding author
}

from Eighteenth Annual Computational Neuroscience Meeting: CNS*2009

Berlin, Germany. 18-23 July 2009

Published: I 3 July 2009

BMC Neuroscience 2009, 10(SuppI I):P75 doi:10.I I86/I47I-2202-I0-SI-P75

This abstract is available from: http://www.biomedcentral.com//47I-2202/I0/SI/P75

(C) $2009 \mathrm{Li}$ and Tang; licensee BioMed Central Ltd.

\section{Background}

The goal of brain functional networks research is to describe the network of function and interactions that describe the cognitive process in the brain. Recently, much effort has been devoted to the reconstruction of the functional networks using a variety of modeling approaches ranging from simple coherence networks through dynamic models. However, most of these models are based on simplistic assumptions. Here we explored a logi-analyzed network, one form of modeling method, to infer directed connectivity among brain regions. This method is based on the assumption that nonlinear interactions among brain regions in terms of their logic relationships play a key role for deciphering the brain networks that underlie the cortex interactions [1]. We formulated a procedure to identify one functional network that is weighted by the high activation level of the regions of interest (ROIs) and receives its direction from the logic relationships captured within the observed fMRI data. This method is used the haemodynamic signals to determine activation or deactivation of ROI. Then, based on a graph coloring scheme and information theory, we identified the brain functional networks that lower and higher order logic interactions of ROIs. A low logic relationship can be described in this way. A region is activated/deactivated only if $B$ region is activated/deactivated, whereas a higher one presents a pattern of activation/deactivation of multiple ROIs. For instance, $\mathrm{C}$ region is activated if and only if A region and $\mathrm{B}$ region are both activated. Next, the probability of each logic pattern in the networks was com- puted as a support level. Finally, a brain functional network was constructed with weights and directionality.

\section{Results and conclusion}

The analysis of functional interactions from a face perception paradigm identified a functional network with directionality and weights that reflects the ROI interactive communication pathways. The proposed method in mining hidden, complicated interactions among brain regions reliably interpreted the experimental results, suggesting that it would be one useful tool for understanding the cognitive system.

\section{Acknowledgements}

This work was supported by NSFC 30670699, NCET-06-0277 and James S. Bower Foundation.

\section{References}

I. Browers PM, Cokus SJ, Eisenberg D, Yeates TO: Use of logic relationships to decipher protein network organization. Science 2004, 304:2246. 